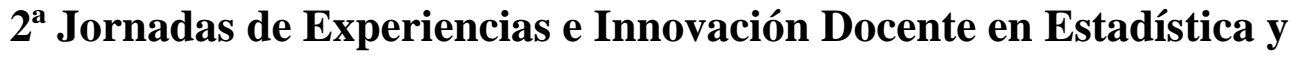 \\ Matemáticas: eXIDO 2017
}

\author{
Miguel Delgado Pineda \\ Universidad Nacional de Educación a Distancia
}

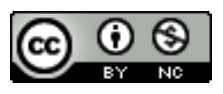

Los días del 17 al 19 de julio de este año, 2017, se ha celebrado en el Centro Asociado de JaénÚbeda (nuevamente en Úbeda) de la UNED el segundo eXIDO: Encuentro de profesores e investigadores en el campo de enseñanza e innovación en Matemáticas a nivel universitario y de Enseñanza Secundaria.

La jornada estuvo coorganizada por la UNED (Centro Asociado y Departamento de Matemáticas Fundamentales), la Universidad Politécnica de Cartagena (Departamento de Matemáticas Aplicadas y Estadística) y la Universidad de Jaén. En esta ocasión ha sido financiado, en parte, por el Centro Asociado y la universidad de Jaén, y en otra parte por la autofinanciación de todos los asistentes, incluyendo a los organizadores.

Se ha mantenido el objetivo principal de este encuentro; compartir experiencias educativas del ámbito cotidiano. En esta ocasión se ha abierto a otros saberes como la Medicina y la realidad virtual. El entorno abierto de profesores interesados en "mejorar" su docencia se ha mantenido si bien predominan la asistencia de profesores de "Matemáticas", tanto universitarios como de Enseñanza Secundaria. El entorno innovador no describir las experiencias dentro de teoría didáctica de la enseñanza de las Matemáticas alguna, simplemente es necesario explicar cuál es el camino experimentado, si sus posibles implicaciones en un futuro próximo. La idea es ver el camino que recorre experimental e innovador que recorren los ponentes de estas jornadas, y tomar conciencia de las numerosas experiencia que no suelen acumularse en otro encuentros de carácter innovador y generalistas.

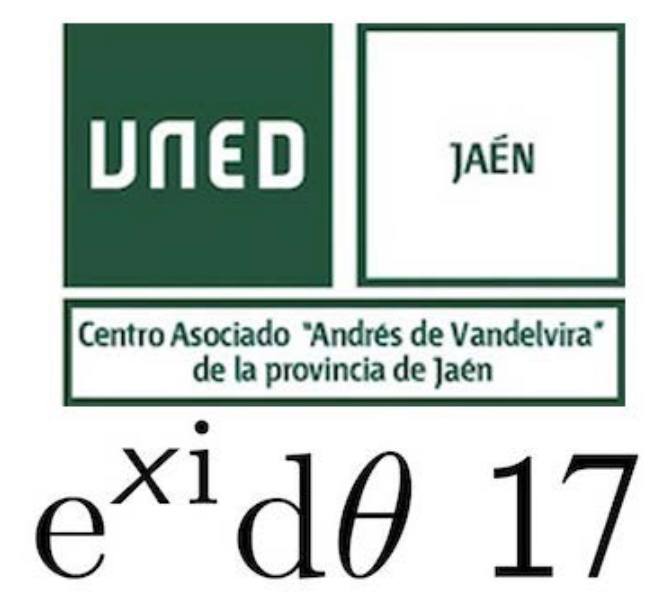

Figura 1: Peculiaridad de logo de las Jornadas

En el diseño de estas jornadas han participado los siguientes miembros de la UNED; Antonio Costa González y Miguel Delgado Pineda (Departamento de. Matemáticas Fundamentales) y Joaquín Arias Vílchez (C. A. Jaén-Úbeda). 
La participación UNED en estas jornadas fue a cargo de Jose Carlos Antoranz Callejo, Beatriz Estrada López, Adoración Medina Albos (tutora) y los mencionados organizadores (Facultad de Ciencias).

- Antonio Costa González Innovaciones en la asignatura Geometría Básica de la UNED.

- Jose Carlos Antoranz Callejo: Matemáticas y Medicina

- Miguel Delgado Pineda: Las rosa reales. Una introducción a los números reales.

- Beatriz Estrada Experiencias docentes en Álgebra Lineal con Maxima: ponte en el lugar del profesor.

- Adoración Medina Albós y Miguel Delgado Pineda: Visualización matemática con Geogebra: Integrales singulares.

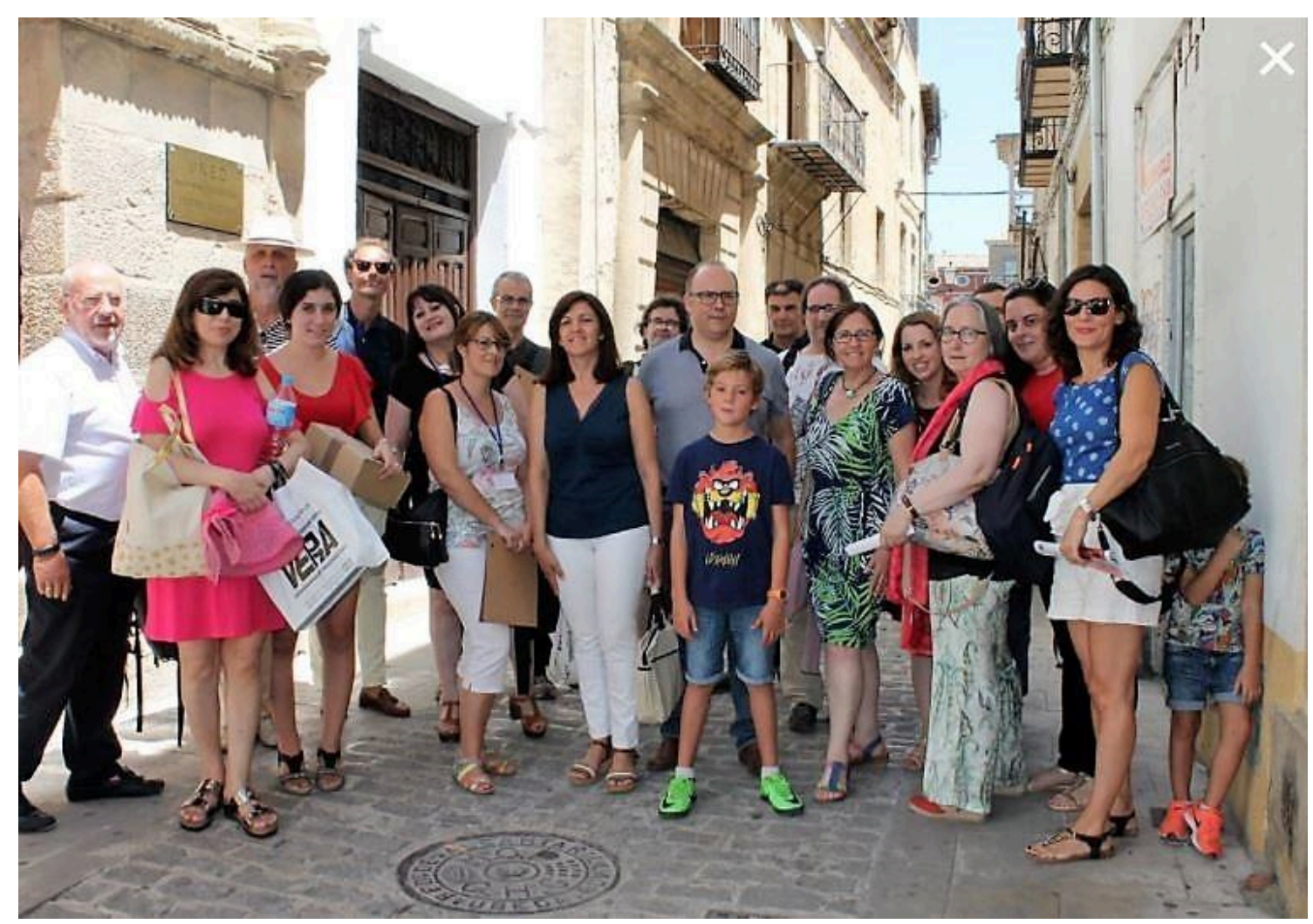

Figura 1: Parte del grupo de asistentes.

$\mathrm{Al}$ igual que las jornadas anteriores, el evento fue inaugurado por: la Concejal de Educación del excelentísimo ayuntamiento de Úbeda, Dña. Elena Rodríguez García, El Director del Centro Asociado Jaén-Úbeda D. Andrés Medina Gómez, el Vicedecano de la Facultad de Ciencias de la UNED: D. Antonio Costa González. y el Secretario de Centro Asociado D. Joaquín Arias Vílchez. 


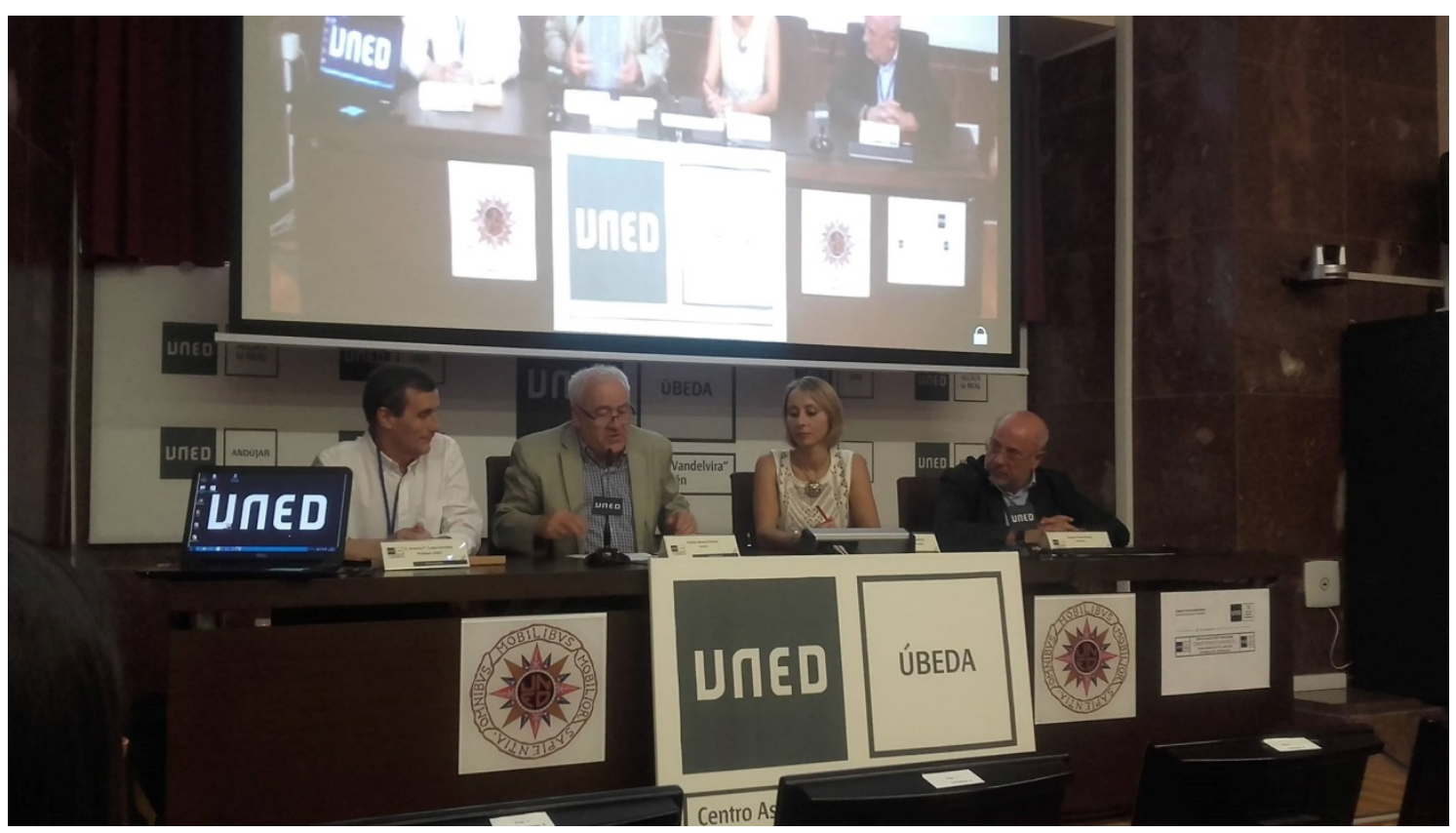

Figura 3: Inauguración de las jornadas.

En esta ocasión se retransmitió en directo la primera sesión íntegramente, vía Internet, a participantes no presenciales. Sin embargo, los retrasos en la vuelta de la información gráfica desaconsejaron seguir por esa línea de comunicación. Quizás en la próxima ocasión las comunicaciones de ida y de retorno sean mejores.

De nuestra Facultad asistieron los siguientes ponentes: Las ponencias fueron las siguientes:

- Antonio Costa González: Experiencias con GeoGebra en la asignatura Geometría Básica del grado de Matemáticas de la UNED.

- Miguel Delgado Pineda: Comunicación icónica y gestual en Análisis Matemático.

- Beatriz Estrada Vídeos docentes para estudiantes del grado de Matemáticas

- Adoración Medina Albos, tutora del Departamento: Enseñar a pensar; del juego al razonamiento matemático. Esta ponencia la realizó junto con Miguel Delgado Pineda. 


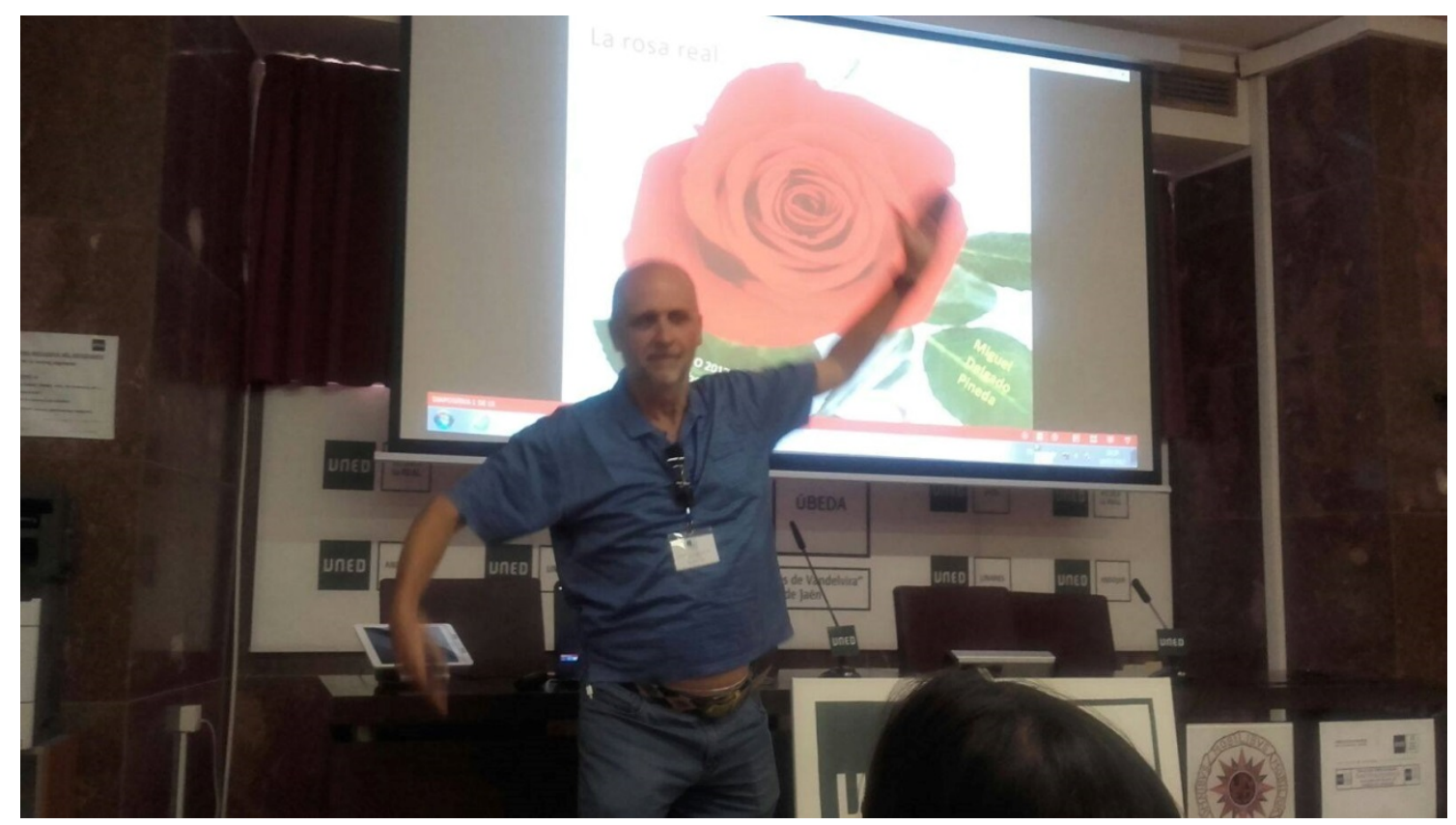

Figura 4: Vista de la ponencia La Rosa Real.

En una última reunión de comité organizador consideró oportuno repetir el próximo año la celebración de las jornadas en su tercera edición, en el mismo lugar: En el Centro Asociado de Úbeda-Jaén sede de Úbeda. Por ello, esperamos que este artículo sirva de llamada a cualquier innovador de sus materias científicas. ¡No duden en acercarte al encuentro eXIDO18! que tendrá lugar a primeros del mes de julio en Úbeda

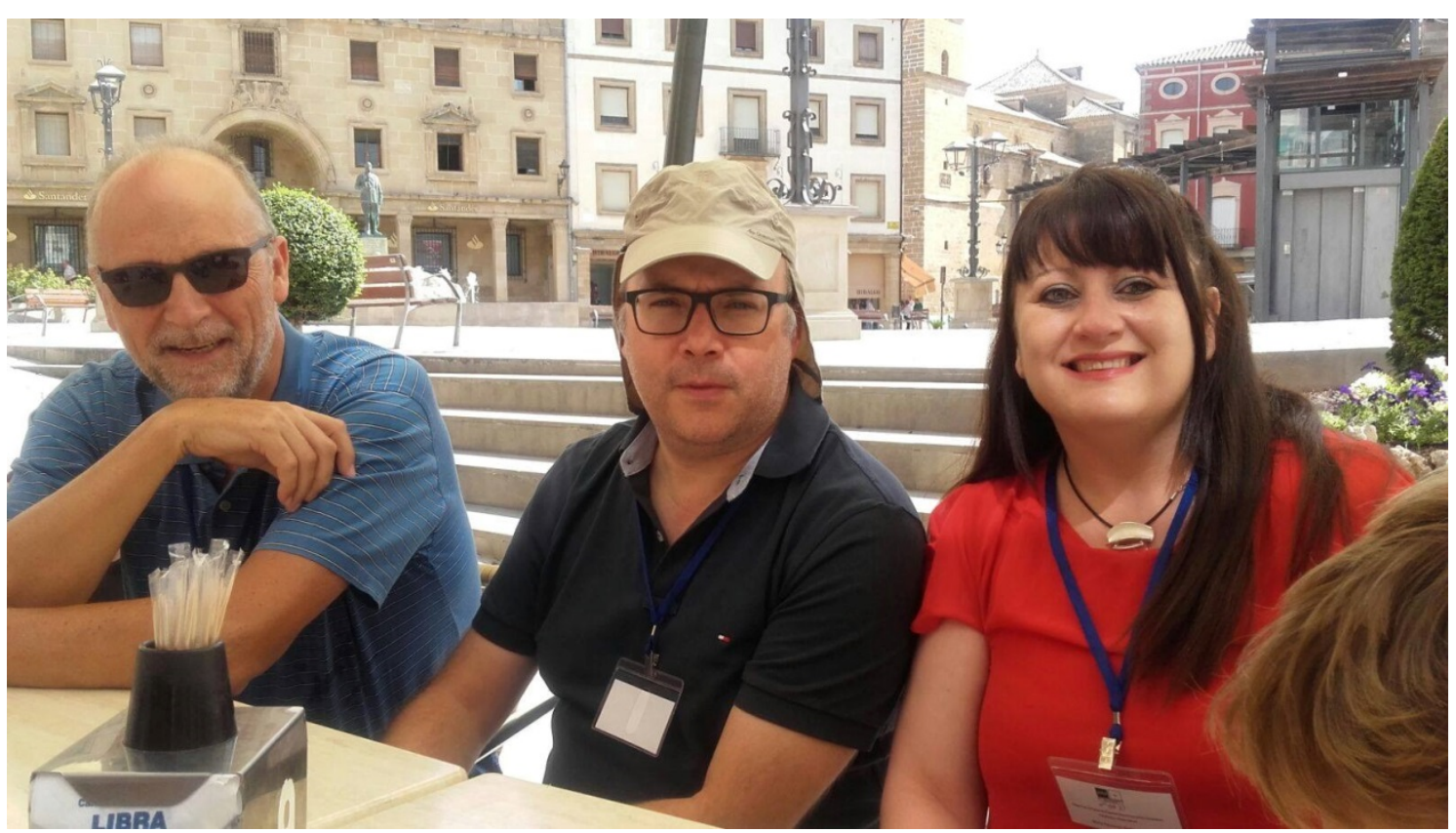

Figura 5: Participantes confraternizando en la plaza de Úbeda. 


\section{Resúmenes de las comunicaciones}

La importancia de la sesión grupal en la clase inversa: Trabajos colaborativos en una asignatura de Matemáticas de Grado durante el curso 2016-2017. Anna Vidal, Francisco J. Boigues and Vicente D. Estruch.

En este trabajo se describen algunos de los trabajos realizados durante el curso 20162017 por los estudiantes del Grado en Ingeniería de Sistemas de Telecomunicación, Sonido e Imagen de la Universitat Politècnica de València, en las prácticas de una asignatura anual del primer año, Matemáticas 2. La utilización de la Clase Inversa en dichas prácticas ha permitido dedicar las horas presenciales a la realización de trabajos colaborativos durante la sesión presencial o grupal. Se ha utilizado la plataforma PoliformaT a través de la cual profesores y estudiantes pueden compartir información sobre sus asignaturas así como utilizar herramientas para su gestión como son tareas, exámenes o la herramienta Lessons que permite la creación de contenidos e itinerarios formativos, facilitándonos la implementación de la clase inversa en esta asignatura. Durante las prácticas nuestros estudiantes han utilizado el programa Matlab consiguiendo entre todos varios productos finales, como una colección de gráficas de curvas en coordenadas paramétricas y polares, una colección de gráficas de superficies y una representación tridimensional de la Sierra de Bernia. Durante el curso se han realizado varios posters de estos resultados conjuntos que han sido expuestos en nuestro aulario, despertando el interés de profesores y estudiantes de otras asignaturas.

\section{Habilidades para que los graduados en matemáticas tengan éxito profesional. Ana M Arnal Pons.}

En este trabajo pretendemos responder a la pregunta: ¿Cómo preparamos a nuestros estudiantes para tener éxito en su fututo? Describimos una serie de habilidades que a nuestro juicio deberían adquirir todos los estudiantes del grado de matemáticas. Enumeramos algunas habilidades básicas como pueden ser un pensamiento adaptivo, capacidad de abstracción, intuición y habilidades tecnológicas. Complementadas por habilidades de investigación, pensamiento crítico, colaboración y empatía, creatividad, comunicación. Justificaremos nuestra propuesta relacionándola con las salidas profesionales de las próximas décadas.

Presentaciones a partir de vídeos y emisiones en directo. Juan Medina Molina.

Después de más de 10 años utilizando vídeos para complementar nuestras clases, seguimos evolucionando para mejorar nuestra labor docente usando este tipo de contenidos. Así, desde hace un tiempo estamos trabajando con un nuevo formato de vídeo, donde en lugar de usar el procedimiento de mano alzada, partimos de un documento o documentos donde incluimos de forma adecuada el texto y expresiones matemáticas, grabamos el archivo de audio, y montamos el vídeo usando el programa Camtasia. En este, vamos descubriendo el documento a la vez que sincronizamos la imagen con la narración, destacando en cada momento los elementos clave. 
En nuestro día a día, nunca hemos realizado reproducciones de vídeo en el aula, en todo caso hemos navegado por el vídeo, añadiendo la explicación en directo. Antes y después de clase, el alumno puede previsualizar o repasar el contenido. Sin embargo, lo que verdaderamente nos interesa para nuestras clases es la presentación.

Con el nuevo formato de vídeo con el que estamos trabajando, resulta muy sencillo y rápido originar una presentación. Lo único que debemos hacer es ir realizando capturas aprovechando la navegación temporal en Camtasia. Así, ahora solo debemos preocuparnos en clase de ir pulsando en el pasador.

¿Qué ganamos con respecto a la creación de una presentación tipo Beamer? En primer lugar, que podemos situar los contenidos de forma flexible, como si lo hiciéramos en la pizarra, con Beamer resulta muy complicado. Por otra parte y fundamental, que el alumno cuenta con los vídeos para trabajar el material por su cuenta, algo que se ha demostrado en los últimos años que le resulta muy útil. Además, este tipo de presentaciones puede ser muy útil para clases online, basta compartir la pantalla mientras realizamos la explicación.

En la parte negativa, la generación de este tipo de vídeos resulta muy costosa, aunque si así lo deseamos, aprovechando el programa Camtasia podríamos realizar la presentación directamente, sin la necesidad de grabar el audio.

Actualmente combinamos el procedimiento "a mano alzada" y este nuevo procedimiento.

Para terminar nuestra presentación, hablaremos sobre las emisiones en directo que llevamos realizando desde hace unos meses en la plataforma YouTube, donde han participado miles de alumnos.

\section{Generación Automática de Pruebas Matemáticas Individualizadas.}

\section{J.Antonio Seijas-Macias.}

La introducción del Espacio Europeo de Educación Superior (EEES) a finales de la década del 2000 supone un importante cambio en el paradigma educativo utilizado en las universidades con docencia presencial. Ahora, el reto de la enseñanza se individualiza y el alumno deja de ser un mero sujeto pasivo para protagonizar el proceso de enseñanza-aprendizaje. En estas circunstancias, se hace necesario un cambio del sistema de gestión de la evaluación de conocimientos mediante la realización de pruebas y trabajos individualizados que permitan reflejar de forma fiel la adquisición de conocimientos por parte del alumnado. En la materia de Matemáticas II correspondiente a la titulación de Grado en Administración y Dirección de Empresas este problema se ve condicionado por la necesidad de contar con medios que permitan la generación y corrección de diferentes pruebas individualizadas con contenidos matemáticos. En este contexto, presentamos la integración mediante las herramientas de LaTeX y Sagemath. 


\section{Algunas experiencias en la evaluación de Estadística en la Universidad de}

Jaén. Sonia Castillo-Gutiérrez, María Dolores Estudillo-Martínez, María Del Pilar Frías-Bustamante and Nuria Ruiz-Fuentes.

El tradicional examen de problemas basado fundamentalmente en realizar cálculos aislados para muestras de datos excesivamente pequeñas y generalmente ajustadas a ciertos criterios y objetivos, queda obsoleto para la evaluación de las competencias que deben adquirir en Estadística los nuevos estudiantes de Grado. El mundo laboral demanda profesionales capaces de manejar y analizar grandes cantidades de datos, seleccionar y aplicar el método apropiado e interpretar adecuadamente los resultados para la toma de decisiones acertadas. En este trabajo explicamos nuestras experiencias en la evaluación de la asignatura Estadística para los Grados de Trabajo Social, Turismo e Ingenierías Industriales. Aportamos métodos de evaluación adaptados a las nuevas exigencias para la formación del alumnado y analizamos las ventajas e inconvenientes en cada uno de los métodos expuestos.

\section{TIC para la mejora del aprendizaje y la adquisición de competencias.} Antonio Miguel Márquez Durán.

En esta comunicación pretendemos mostrar cómo las TIC (Tecnologías de la Información y de la Comunicación) nos pueden proporcionar una nueva forma de presentar, de trasmitir el conocimiento a nuestros alumnos, una forma más atractiva, más interactiva y más acorde con los nuevos tiempos y las nuevas tecnologías. En concreto realizaremos una comparativa entre el material tradicional de una asignatura concreta, Estadística Empresarial I, del Grado en Administración y Dirección de Empresas, y cómo quedaría con el uso de Wimba Create. Todo ello dentro del marco de un proyecto de innovación que pretende demostrar que este tipo de herramientas contribuye también a la mejora del aprendizaje autónomo y a la adquisición de competencias entre nuestros alumnos.

Las rosas reales: Una introducción a los números reales. Miguel Delgado Pineda.

La imagen que se asocia habitualmente al conjunto de los números reales con una línea recta real para consolidar lo que llamamos recta real, esconde de forma sutil el concepto de número real y más concretamente el de número irracional. Si bien en cierta época de la Grecia Clásica se sabía de la existencia de algunos números reales, por sin estructura, los números se trataban como medida de las características de los objetos o figuras. Se trataba con números (racionales) conmensurables y se dejaba en el ostracismo a otros números (irracionales) incomensurables. Era evidente que para algunas figuras, por ejemplo un cuadrado de lado unitario, se podía medir su área, pero no podían medirse la longitud de la diagonal. Resulta que en la enseñanza de Matemáticas esto mismo sucede de una forma solapada, por ello en el estudiante se inician todo un conjuntos de dificultades didácticas que le impiden dimensionar la grandeza de la estructura del número real. 
En esta presentación aportamos una aproximación al concepto de número real coherente con la "medibilidad" de los números racionales e irracionales haciendo una aproximación basada en la Paradoja de Aquiles y la tortuga, y en bipartición de un segmento rectilíneo.

La situación biparticional puede ser presentada con otros objetos geométricos y ser modelados mediante notación numérica binaria. Esto nos permite introducir las rosas reales como elemento visual que genera de forma clara la imagen mental que es necesaria para tratar con los números reales y su estructura. Así pues el modelo numérico emerge como una simplificación del objeto, en lugar de que el método numérico preceda a los objetos.

Esta introducción ha sido tratada en cursos de formación de profesorado y en los másteres de Enseñanza Secundaria de la UNED.

Magnitudes, principios y ecuaciones como base en la enseñanza del Cálculo. Daniel Cardenas-Morales.

Esta comunicación pretende dar cuenta de la experiencia docente que durante años se ha llevado a cabo para enseñar Cálculo a estudiantes universitarios de primer año en carreras de ciencias experimentales. Sobre la base que representan los principios naturales y el conocimiento somero de las principales magnitudes se da cuerpo a algunas nociones fundamentales propias del Análisis Matemático. Se presentan algunas ventajas e inconvenientes asociados a este enfoque.

\section{Evaluación continua, clase inversa y cooperación activa en Matemáticas}

para ingenieros. Luis Manuel Sánchez Ruiz, Sergio Blanes, Maria Teresa Capilla, María Belén García Mora, Santiago Moll-Lopez, Jose Antonio Moraño and M.-Dolores Roselló.

La Universitat Politècnica de Valencia (UPV) facilita la creación de equipos de innovación y calidad educativa (EICE). Uno de dichos equipos es GRIM4E (GRoup of Innnovative Methodologies and Assessment For Engineering education) que comenzó a realizar innovaciones metodológicas al adaptar las asignaturas de matemáticas a los grados surgidos dentro del proceso de Bolonia. Así, en la titulación de Ingeniería Aeroespacial de la UPV se pasó de tener cuatro asignaturas de contenido matemático con una carga lectiva de 36 créditos a tres con una carga de 24 créditos. Algunas de las innovaciones ya habían sido iniciadas con anterioridad como una evaluación continua de todas las actividades de aprendizaje desarrolladas durante el curso, con más de 30 actos de evaluación en la actualidad en las asignaturas anuales y 10 en las semestrales. Otras fueron pioneras como el empleo sistemático de la clase inversa en las prácticas informáticas de las asignaturas involucradas. Una innovación reciente destacada en nuestro ámbito ha sido el fomento de una actitud activa y colaborativa de los alumnos en la preparación de los actos de evaluación más relevantes.

En esta comunicación presentamos estas líneas desarrolladas por GRIM4E e incluimos 
resultados de encuestas anónimas realizadas para recabar la percepción de los alumnos sobre la metodología mixta empleada.

Usando Matlab como complemento de mis clases. Juan Carlos Trillo Moya.

En esta charla describiremos el uso de algunas interfaces gráficas de Matlab como apoyo docente en algunas asignaturas de matemáticas en Ingeniería.

Experiencias docentes en Álgebra Lineal con Maxima: ponte en el lugar del profesor. Beatriz Estrada.

Son muchos los motivos por los que resulta interesante tanto para un estudiante como para un profesor de Matemáticas saber utilizar un programa de cálculo simbólico.

En las asignaturas Álgebra Lineal I y II del Grado en Matemáticas de la UNED propongo a los estudiantes el uso del conocido software libre: Maxima. Para incentivar su uso reciben unos tutoriales y se informa a los estudiantes de que les sirve para comprobar si han resuelto bien los ejercicios y para la experimentación.

En efecto, la fase experimental del estudio de las Matemáticas cobra un impulso enorme cuando se dispone de un programa de cálculo.

El objetivo de la comunicación es presentar una experiencia docente basada en un tipo distinto de ejercicios para los cuales es necesario el uso de un programa de cálculo simbólico. La propuesta, básicamente, podría resumirse en una frase dirigida al estudiante: "ponte en el lugar del profesor".

Innovaciones en la asignatura Geometría Básica de la UNED. Antonio Félix Costa.

La asignatura Geometría Básica es del primer curso del Grado en Matemáticas de la UNED. En esta comunicación presentamos las innovaciones docentes que se han llevado a cabo este año en la asignatura, en particular el uso de Geogebra y de vídeos.

Visualización Matemática con Geogebra: Integrales Impropias. Miguel Delgado and María Adoración Medina:

Presentamos un proyecto de Innovación Docente que utiliza el software de Geometría Dinámica Geogebra para facilitar el aprendizaje de las integrales impropias a los estudiantes de primer curso del Grado de Ciencias Matemáticas en la UNED. Para ello se ha desarrollado una ingeniería visual, que consta de diversos laboratorios de simulación matemática. Estos laboratorios van más allá de la simple representación dinámica de problemas y conceptos. Están específicamente diseñados para incidir en el proceso de Visualización Matemática de los estudiantes, entendiéndose este como el proceso de codificación de información matemática en una imagen. Este proyecto ha sido desarrollado en el marco de las acciones del Grupo de Innovación Docente (GID) del Departamento de Matemáticas Fundamentales de la Facultad de Ciencias de UNED, $\pi$-Mat. 